\title{
On the Geometry of the Conic and Triangle.
}

\author{
By John Miller.
}

(Read March 19th. Received, June 1st, 1908.)

$\$ 1$.

In the Proceedings of 1905-6 Mr Pinkerton gave an extension of the nine point circle to a nine point conic. This raises the question of the extension of the geometry of the circle and triangle to that of the conic and triangle. If a triangle with its associated system of lines and circles be orthogonally projected on a second plane we have a triangle with an associated system of lines and homothetic ellipses. Pairs of perpendicular lines are projected into lines parallel to pairs of conjugate diameters. Such lines will be called, for shortness, in the sequel, conjugate lines. In any relation between lengths of lines, these lengths will be replaced by their ratios to the lengths of the parallel radii of one of the homothetic ellipses.

The question now arises whether this geometry of the triangle holds when instead of this system of homothetic ellipses we have hyperbolas. On projecting a circle into a hyperbola, pairs of perpendicular lines do not project into pairs of conjugate lines, for the lines perpendicular to a given line project into concurrent lines. Further, the circles of a plane are not all projected into hyperbolas, much less into homothetic hyperbolas.

Nevertheless, the corresponding geometry exists. The present paper is an attempt to indicate by geometric proofs the point of continuity with the proofs for the geometry of the triangle and circle. The amount of material is very large so that only some excerpts are given and the proofs of some theorems which the extension suggests are omitted to shorten the paper.

A system of homothetic conics has two real or imaginary common points at infinity. A hyperbola and its conjugates will be considered homothetic. Thus circles have in common the two circular points at infinity. Each conic, therefore, of the system is determined by three points in the finite part of the plane. This is the first principle on which the geometry depends. 
We have next the theorem that the ratio of the products of the segments of two chords or secants of a conic through any point is equal to the ratio of the squares on parallel radii. For the circle this ratio is unity, and we have similar triangles bringing in equality of angles. Also, since all the radii of a circle are equal, angles in the same segment are equal. The proofs of the geometry of the circle and triangle generally depend on using relations between angles. It will be seen that in the extension to conics the proofs usually involve the theorem concerning the segments of two chords or secants.*

\section{$\S 2$.}

\section{"Orthocentre." Nine Point Conic.}

Let $O$ (Fig. 10) be the centre of a circumconic of a triangle $\mathrm{ABC} ; \mathrm{U}, \mathrm{V}, \mathrm{W}$ the mid points of the sides; $\mathrm{AD}, \mathrm{BE}, \mathrm{CF}$ parallel to $\mathrm{OU}, \mathrm{OV}, \mathrm{OW} ; a_{1}^{2}, b_{1}^{2}, c_{1}^{2}$ the squares of the radii parallel to $a, b, c$.

Since BE, CE; CF, BF are pairs of conjugates, a homothetic conic with $\mathrm{BC}$ as diameter passes through $\mathrm{E}$ and $\mathrm{F}$.

$$
\therefore \frac{\mathrm{AF} \cdot c}{\mathrm{AE} \cdot b}=\frac{c_{1}^{2}}{b_{1}^{2}} . \quad \text { Similarly } \frac{\mathrm{BD} \cdot a}{\mathrm{BF} \cdot c}=\frac{a_{1}^{2}}{c_{1}^{2}} \text { and } \frac{\mathrm{CE} \cdot b}{\mathrm{CD} \cdot a}=\frac{b_{1}^{2}}{a_{1}^{2}} \text {. }
$$

Hence by Ceva's theorem AD, BE, CF intersect in a point $H$. From the first equation

$$
\begin{aligned}
& \frac{(c-\mathrm{BF}) c}{c_{1}{ }^{2}}=\frac{(b-\mathrm{CE}) b}{b_{1}{ }^{2}} \\
& \frac{b \cdot \mathrm{CE}}{b_{1}{ }^{2}}-\frac{c \cdot \mathrm{BF}}{c_{1}{ }^{2}}=\frac{b^{2}}{b_{1}{ }^{2}}-\frac{c^{2}}{c_{1}{ }^{2}} .
\end{aligned}
$$

or

- I am indebted to Dr Muirhead for reference to a paper by L. Ripert (La Dualité et l'Homographie dans le Triangle et le Tétrahedre, Paris, Gauthier-Villars et Fils, 1898.) In this short generalising paper M. Ripert has anticipated some of my fundemental ideas. His work is, however, very different, being mainly an application of barycentric co-ordinates. I may state, that in the detailed working out of results, I often used proofs by trilinear and barycentric co-ordinates, but discarded them for proofs involving the direct application of the principles already mentioned. 
From the second by transposing and adding

$$
\begin{gathered}
\frac{b \cdot \mathrm{CE}}{b_{1}{ }^{2}}+\frac{c \cdot \mathrm{BF}}{c_{1}{ }^{2}}=\frac{a^{2}}{a^{2{ }^{2}}} . \\
\therefore \frac{2 b \cdot \mathrm{CE}}{b_{1}{ }^{2}}=\frac{2 a \cdot \mathrm{CD}}{a_{1}{ }^{2}}=\frac{a^{2}}{a_{1}{ }^{2}}+\frac{b^{2}}{b_{1}{ }^{2}}-\frac{c^{2}}{c_{1}^{2}}, \text { etc. }
\end{gathered}
$$

We give a second simple proof.

Let $\quad \mathrm{XOY}^{\prime}$ parallel to $c$ cut $a$ in $\mathrm{X}$ and $b$ in $\mathrm{Y}^{\prime}$;

$\mathrm{X}^{\prime} \mathrm{O} Z \mathbf{Y}$ parallel to $b$ cut $a$ in $\mathrm{X}^{\prime}$ and $c$ in $Z$;

$\mathrm{Z}^{\prime} \mathrm{OY}$ parallel to $a$ cut $c$ in $\mathrm{Z}^{\prime}$ and $b$ in Y. (Fig. 10.)

Since $\frac{\mathrm{XU}}{\mathrm{UX}^{\prime}}=\frac{\mathrm{BD}}{\mathrm{DC}}$ it is to be proved that

$$
\frac{\mathrm{XU}}{\mathrm{UX^{ \prime }}} \cdot \frac{\mathrm{YV}}{\mathrm{VY^{ \prime }}} \cdot \frac{\mathrm{ZW}}{\mathrm{WZ}}=1
$$

Now

$$
\begin{gathered}
\frac{\mathrm{AZ}}{c}=\frac{\mathrm{AY}}{b} \text { or } \frac{\frac{1}{2} c+\mathrm{WZ}^{\prime}}{c}=\frac{\frac{1}{2} b+\mathrm{VY}}{b} . \\
\therefore \frac{\mathrm{WZ}^{\prime}}{\mathrm{VY}}=\frac{c}{b} . \quad \therefore \text { etc. }
\end{gathered}
$$

The proof for the nine point conic now proceeds as in $\mathrm{Mr}$ Pinkerton's paper.

Let (Fig. 10) $\mathrm{AO}$ and $\mathrm{AD}$ meet the circumconic again in $O^{\prime}$ and $D^{\prime}$. $O^{\prime} D^{\prime}$ is parallel to $U D$. If $O U$ meet $O^{\prime} D^{\prime}$ in $U^{\prime}$, $O U^{\prime}=\frac{1}{2} A^{\prime}$. But $\mathrm{OU}=\frac{1}{2} \mathrm{AH} . \quad \therefore \mathrm{HN}=\mathrm{DD}^{\prime}$.

Let $p^{2}$ be the square of the radius parallel to $\mathrm{AD}$.

$$
\frac{\mathrm{AD} \cdot \mathrm{DD}^{\prime}}{p^{2}}=\frac{\mathrm{AD} \cdot \mathrm{HD}}{p^{2}}=\frac{\mathrm{BD} \cdot \mathrm{DC}}{a_{1}{ }^{2}}-\left(\frac{a^{2}}{a_{1}{ }^{2}}+\frac{b^{2}}{b_{1}{ }^{2}}-\frac{c^{2}}{c_{1}{ }^{2}}\right)\left(\frac{a^{2}}{a_{1}{ }^{2}}-\frac{b^{2}}{b_{1}{ }^{3}}+\frac{c^{2}}{c_{1}{ }^{2}}\right) \div \frac{2 a^{2}}{a_{1}{ }^{2}} \text {. }
$$

Also a homothetic conic with $\mathrm{CH}$ as diameter passes through $\mathrm{D}, \mathrm{E}$.

$$
\begin{aligned}
& \therefore \frac{2 \mathrm{AH} \cdot \mathrm{AD}}{p^{2}}=\frac{2 b \cdot \mathrm{AE}}{b_{1}{ }^{2}}=\frac{b^{2}}{b_{1}{ }^{2}}+\frac{c^{2}}{c_{1}{ }^{2}}-\frac{a^{2}}{a_{1}{ }^{2}} . \\
& \therefore \frac{2 \mathrm{AD}^{2}}{p^{2}}-\frac{2 \mathrm{AD} \cdot \mathrm{HD}}{p^{2}}=\frac{b^{2}}{b_{1}{ }^{2}}+\frac{c^{2}}{c_{1}{ }^{2}}-\frac{a^{2}}{a_{1}{ }^{2}} .
\end{aligned}
$$


$\therefore \frac{4 a^{2} \mathrm{AD}^{2}}{p^{2} a_{1}^{2}}=\mathrm{S}^{2}=s\left(s-\frac{a}{a_{1}}\right)\left(s-\frac{b}{b_{1}}\right)\left(s-\frac{c}{c_{1}}\right)$ where $2 s=\frac{a}{a_{1}}+\frac{b}{b_{1}}+\frac{c}{c_{1}}$.

It may be noted that if the conic be a hyperbola the results are still real since a radius enters by its square only. These results show that extended trigonometrical or hyperbolic sines or cosines might be used with advantage. An angle will have to be supposed given only when the directions of both arms are given. If a homothetic conic be drawn with the intersection as centre and from the end of one arm the conjugate to the second arm be drawn, the sine will be the ratio of this ordinate to the parallel radius and the cosine will be the ratio of the abscissa to the parallel radius.

\section{$\$ 3$.}

“W Allace Line."

Let $P$ be a point on the circumconic and $P Q, P R, P S$ conjugate to $\mathrm{BC}, \mathrm{CA}, \mathrm{AB}$. (Fig. 11).

Since $\mathbf{P Q}, \mathrm{CQ}$ are conjugate and $\mathrm{PR}, \mathrm{CR}$ are conjugate, the homothetic conic on $P C$ as diameter passes through $Q, R$. Let $P Q, P R, P S$ cut the circumconic in $Q_{1}, R_{1}, S_{1}$. The circumconic, the conic PCQR and the pair of lines $P Q, C R$ have a common chord CP; therefore their other three chords meet in a point. The common chord of the two homothetic chords is at infinity and therefore $\mathrm{QR}, A Q_{1}$ are parallel. Similarly taking the pair of lines $\mathrm{CQ}, \mathrm{PR}$ we find that $\mathrm{QR}$ and $\mathrm{BR}_{1}$ are parallel. $\mathrm{By}$ considering the quadrilateral $P R A S$ we find that $R S$ and $B R_{1}$ are parallel and therefore $Q, R, S$ are collinear.

If $\mathrm{H}_{1}$ be taken on $\mathrm{QP}$ such that $\mathrm{QH}_{1}=\mathrm{Q}_{1} \mathrm{Q}, \mathrm{H}_{1}$ is the "orthocentre" of the triangle $\mathrm{PBC}$ and $\mathrm{PH}_{1}=2 \mathrm{OU}=\mathrm{AH}$. Let the "Wallace line" meet $\mathrm{AD}$ in $\mathrm{X}$ (Fig. 11); $\mathrm{QQ}_{1} \mathrm{AX}$ is a parallelogram and $Q_{1} Q=A X$. Therefore $Q P=H X$ and so the "Wallace line" bisects $\mathrm{PH}$ in $\mathrm{M}$. which is a point on the nine point conic.

Let $\mathrm{N}$ be the centre of the nine point conic and $\mathrm{G}$ the centroid. Let $G P$ cut $O M$ in $G_{1}$ and draw $G_{G_{2}}$ parallel to NMI to cut $O M$ in $\mathrm{G}_{2}$, (Fig. 12). 
Since $\mathrm{OG}=\frac{2}{3} \mathrm{ON}, \mathrm{GG}_{2}=\frac{2}{3} \mathrm{NM}$,

$$
\frac{P G_{1}}{G_{1} G}=\frac{O P}{G_{G_{2}}}=\frac{2 N M}{\frac{2}{3} N M}=\frac{3}{1} .
$$

$\therefore G_{1}$ is the centre of mean position of $A, B, C, P$.

Also

$$
\begin{gathered}
\frac{\mathrm{OG}_{1}}{\mathrm{G}_{1} \mathrm{G}_{2}}=\frac{3}{1} \text { and } \mathrm{OG}_{2}=\frac{2}{3} \mathrm{OM} . \\
\therefore O \mathrm{OG}_{1}=3\left(\mathrm{OG}_{2}-O \mathrm{OG}_{1}\right)=2 \mathrm{OM}-3 O \mathrm{G}_{1}=\frac{1}{2} \mathrm{OM} .
\end{gathered}
$$

$\therefore \mathrm{M}$ is got by joining the centre of the circumconic to the centre of mean position of the four points and producing this line its own length. The symmetry shows that the four "Wallace lines" obtained by taking three of the points as vertices and the fourth as the point from which the conjugates are drawn, all meet in a point.

Any transversal of a triangle can be regarded in an infinite number of ways as a "Wallace line." Draw (Fig. 11) QP arbitrarily and let the parallel to QP through $\mathrm{A}$ cut the transversal in $\mathrm{X}$. Bisect $Q X$ in $M$. We have then five points $U, V, W, D, M$ on the nine point conic. These determine it and the homothetic circumconic.

The locus of $P$ for a given transversal is a straight line. The locus of the "orthocentre" $H$ is a hyperbola through $A, B, C$ with asymptotes parallel to QRS and the locus of $P$. The locus of the centre $O$ is a hyperbola passing through $U, V, W$ and with asymptotes parallel to the same lines.

If $\mathrm{P}, \mathrm{A}, \mathrm{B}, \mathrm{C}$ be given the envelope of the "Wallace line" of $P$ is a conic touching the sides $A B, B C, C A$. The loci of $\mathrm{M}, \mathrm{N}, \mathrm{H}$ are conics, etc.

$$
\S 4 \text {. }
$$

\section{Conics Touching the Sides.}

Four conics homothetic to the circumconic can be drawn to touch the sides unless the circumconic is a hyperbola and the 
vertices are not all on the same branch; in this case the tangent conics are imaginary. Draw the diameters of the circumconic conjugate to the sides $\mathrm{BC}, \mathrm{CA}, \mathrm{AB}$ and draw tangents at their ends. These form in all eight triangles similar to $\mathrm{ABC}$, but four are equal to the remaining four. We give a figure (Fig. 13) for the ellipse only. In this figure $A_{1} B_{1} C_{1}$ and the conic correspond to $\mathrm{ABC}$ and the inscribed ellipse: $\mathrm{A}_{1} \mathrm{~B}_{2} \mathrm{C}_{2}$ and the conic correspond to $\mathrm{ABC}$ and the ellipse touching $\mathrm{BC}$ externally. The centres $I, I_{1}, I_{2}, I_{3}$ can now be easily found for $O$ say lies with respect to $A_{1}, B_{2}, C_{2}$ as $I_{1}$ with respect to $A, B, C$.

$A, I, I_{1}$ are collinear and $I_{2}, A, I_{3}$.

$\mathrm{OA}_{1}$ and $\mathrm{OA}_{2}$ are conjugate diameters. Therefore $\mathrm{AII}_{1}$ and $I_{2} A_{3}$ are conjugate or $A B C$ is the "pedal " triangle of $I_{1} I_{2} I_{3}$ and $I$ the "orthocentre." The circumconic of $\mathrm{ABC}$ is the nine point conic of $I_{1} I_{2} I_{3}$ and the points $K$ and $L$ say where it meets $I_{2} I_{3}, I I_{1}$ again are the mid points of $I_{2} I_{3}$ and $I I_{1}$.

Since $\mathrm{KA}$ and LA are conjugate, $\mathrm{KL}$ is a diameter. Also since the mid points of the diagonals of the quadrilateral $\mathrm{BI}_{1} \mathrm{CI}$ are collinear, $\mathrm{KL}$ bisects $\mathrm{BC}$ in $\mathrm{U}$ and is therefore parallel to the radii $r, r_{1}, r_{2}, r_{3}$ drawn from $\mathrm{I}, \mathrm{I}_{1}, \mathrm{I}_{2}, \mathrm{I}_{3}$ to the points of contact of the conics with BC.

If $\mathrm{KL}=2 \mathrm{R}, r_{2}+r_{3}=2 \mathrm{KU}$ and $r_{1}-r=2 \mathrm{UL}$. Therefore if $r, r_{1}, r_{2}, r_{3}, \mathrm{R}$ be any parallel radii

$$
r_{1}+r_{2}+r_{3}-r=4 \mathrm{R} \text {. }
$$

The preceding results give a method of inscribing in a conic a triangle whose sides will be parallel to those of a given triangle. Draw three chords parallel to the sides and find their diameters. Through three of the ends of the diameters draw tangents giving a triangle circumseribed to the conic and similar to the given triangle. Through the point of contact of a side draw a parallel to the line joining the opposite vertex to the centre. The three lines thus drawn meet the conic again in the vertices of the required triangle.

If the inscribed ellipse or corresponding hyperbola touch $\mathrm{AB}$ in $\mathrm{N}$ and $\mathrm{AC}$ in $\mathrm{M}$, then $\frac{\mathrm{AM}^{2}}{b_{1}{ }^{2}}=\frac{\mathrm{AN}^{2}}{c_{1}{ }^{2}}$, etc. 
Hence $\quad \frac{A \mathrm{M}}{b_{1}}=\frac{\mathrm{AN}}{c_{1}}=\frac{1}{2}\left(\frac{b}{b_{1}}+\frac{c}{c_{1}}-\frac{a}{a_{1}}\right)=s-\frac{a}{a_{1}}$.

Similarly we can find the other segments.

Let $I$ be the centre of the inscribed ellipse or corresponding hyperbola and let $r$ and $R$ be the radius of the inconic along AI and of the circumconic parallel to AI.

$$
\begin{gathered}
\frac{(\mathrm{AI}-r)(\mathrm{AI}+r)}{\mathrm{R}^{2}}=\frac{\mathrm{AN}^{2}}{c_{1}{ }^{2}}=\left(s-\frac{a}{a_{\mathfrak{l}}}\right)^{2} \\
\therefore \frac{\mathrm{AI}^{2}}{\mathrm{R}^{2}}=\left(s-\frac{a}{a_{1}}\right)^{2}+\frac{r^{2}}{\mathrm{R}^{2}}
\end{gathered}
$$

Similarly

$$
\frac{\mathrm{AI}_{1}{ }^{2}}{\mathrm{R}^{2}}=s^{2}+\frac{r_{1}{ }^{2}}{\mathrm{R}^{2}}
$$

Also

$$
\begin{aligned}
\frac{\mathrm{AI}}{\mathrm{AI}_{1}=\frac{s-\frac{a}{a_{1}}}{s} \text { and } \frac{r_{1}}{r}} & =\frac{4 \mathrm{R}}{r}+\mathrm{I}-\frac{r_{3}}{r}-\frac{r_{3}}{r} \\
& =\frac{4 \mathrm{R}}{r}+I-\frac{s}{s-\frac{b}{b_{1}}}-\frac{s}{s-\frac{c}{c_{1}}} .
\end{aligned}
$$

From these the ratio of similarity $\frac{r}{R}$ is found to be

$$
\frac{4\left(s-\frac{a}{a_{1}}\right)\left(s-\frac{b}{b_{1}}\right)\left(s-\frac{c}{c_{1}}\right)}{\frac{a b c}{a_{1} b_{2} c_{1}}} .
$$

"Isogonal Conjugates."

If $\mathrm{AO}, \mathrm{AO}_{1}$ are two lines such that when the conjugates $\mathrm{ON}, \mathrm{O}_{1} \mathrm{~N}_{1}$ are drawn to $\mathrm{AB}$ and the conjugates $\mathrm{OM}, O \mathrm{M}_{1}$ to $\mathrm{AC}$ 


$$
\frac{\mathrm{ON} \cdot \mathrm{O}_{1} \mathrm{~N}_{1}}{r^{2}}=\frac{\mathrm{OM} \cdot \mathrm{O}_{1} \mathrm{M}_{1}}{q^{2}}
$$

where $p, q, r$ are the radii conjugate to $a, b, c$, then $\mathrm{AO}, \mathrm{AO}_{1}$ will be called " isogonally conjugate."

If three lines from the vertices are concurrent their "isogonal conjugates" are concurrent. Let $O, O_{1}$ be the points of concurrency called "isogonal conjugate" points. If the circumconic is a hyperbola and $A$ is on a different branch from $B$ and $C, O$ and $O_{1}$ are on the same side of $\mathrm{AB}$ or $\mathrm{AC}$ but on different sides of $\mathrm{BC}$.

If $\mathrm{OL}, \mathrm{O}_{1} \mathrm{~L}_{1}$ be conjugate to $\mathrm{BC}$ the six points $\mathrm{I}, \mathrm{L}_{2}, \mathrm{M}, \mathrm{M}_{1}$, $N, N$, lie on a homothetic conic whose centre is $P$ the mid point of $\mathrm{OO}_{1}$. Take the homothetic conic with centre $\mathrm{P}$ and passing through $\mathrm{L}$. It will pass through $\mathrm{L}_{1}$ and by a little indirect work can be proved to pass through $M, M_{1}, N, N_{1}$.

If the conjugates $\mathrm{AD}, \mathrm{BE}, \mathrm{CF}$ to the sides meet the circumconic in $D_{1}, E_{1}, F_{1}$ then

$$
\frac{\mathrm{AH} \cdot \mathrm{HD}_{1}}{\mathrm{BH} \cdot \overline{\mathrm{HE}}}=\frac{p^{2}}{q^{2}} .
$$

But $\mathrm{AH}=2 \mathrm{OU}, \quad \mathrm{BH}=2 \mathrm{OV}, \quad \mathrm{HD}_{1}=2 \mathrm{HD}, \quad \mathrm{HE}_{1}=2 \mathrm{HE}$; $\therefore$ the circumcentre $O$ and the "orthocentre" $H$ are "isogonally conjugate."

$$
\S 6 .
$$

\section{"Antiparallels."}

A line MN parallel to the tangent at $\mathbf{A}$ is "antiparallel" to BC. Let $\mathrm{MN}$ cut $\mathrm{AC}$ in $\mathrm{M}$ and $\mathrm{AB}$ in $\mathrm{N}$. Consider the homothetic conic through $\mathrm{B}, \mathrm{C}, \mathrm{M}$. This, the circumconic and the lines $A B, A C$ have the common chord BC. Their other three chords then are concurrent. Hence the second chord of the conic BCM and the lines $\mathrm{AB}, \mathrm{AC}$ is parallel to the tangent at $\mathrm{A}$. This conic then passes through $\mathrm{N}$.

The sides of the "pedal" triangle are therefore parallel to the tangents at the vertices.

. Let $P, Q, R$ be the poles of the sides with regard to the circumconic. Draw the "antiparallel" to $B C$ through $P$ meeting $A B$ in $\mathrm{N}$ and $\mathrm{AC}$ in $\mathrm{M}$. 
The triangles PCM, QCA are similar and the triangles NBP, ABR.

$$
\begin{aligned}
& \therefore \frac{P M}{P C}=\frac{A Q}{C Q} \text { and } \frac{N P}{\overline{B P}}=\frac{R A}{R B} \\
& \therefore \frac{N P}{P M}=\frac{R A}{A Q} \cdot \frac{Q C}{C P} \cdot \frac{P B}{B R}=1
\end{aligned}
$$

since $\mathrm{AP}, \mathrm{BQ}, \mathrm{CR}$ are concurrent.

$\therefore \mathrm{NP}=\mathrm{PM}$ and the bisectors $\mathrm{AP}, \mathrm{BQ}, \mathrm{CR}$ of the "antiparallels" meet in the "symmedian" centre $\mathrm{K}$. The locus of the "symmedian" centre $K$ of the triangle $A B C$ for all conics passing through the four points $\mathrm{Z}, \mathrm{A}, \mathrm{B}, \mathrm{C}$ is the polar of the point $\mathrm{Z}$ with respect to the triangle $\mathrm{ABC}$.

$$
\begin{gathered}
\$ 7 . \\
\text { "Cosine" Conic }
\end{gathered}
$$

Through the "symmedian point" $\mathrm{K}$ let the antiparallel $\mathrm{YZ} \mathbf{Z}_{1}$ to $B C$ be drawn cutting $A C$ in $Y$ and $A B$ in $Z_{1}$ (Fig. 14.) Similarly let the antiparallels $Z X_{1}, X Y_{1}$ to $C A$ and $A B$ cut $B C$ in $X$ and $X_{1}$, $\mathrm{AB}$ in $\mathrm{Z}$ and $\mathrm{AC}$ in $\mathrm{Y}_{1}$.

Then

$$
\mathrm{ZK}=\mathrm{KX}_{1}, \mathrm{Z}_{1} \mathrm{~K}=\mathrm{KY}, \mathrm{XK}=\mathrm{KY} \text {. }
$$

$\therefore \mathrm{ZY}_{1}$ is parallel to $\mathrm{BC}, \mathrm{Z}_{1} \mathrm{X}$ to $\mathrm{AC}$ and $\mathrm{X}_{1} \mathrm{Y}$ to $\mathrm{AB}$.

$$
\begin{gathered}
\therefore \frac{\mathrm{AZ}}{\overline{\mathrm{AY}} Y_{1}}=\frac{c}{b}, \frac{\mathrm{BX}}{\mathrm{BZ}}=\frac{a}{c}, \frac{\mathrm{CY}}{\mathrm{CX}_{1}}=\frac{b}{a} . \\
\therefore \frac{\mathrm{AZ} \cdot \mathrm{AZ}_{1} \cdot \mathrm{BX} \cdot \mathrm{BX}_{1} \cdot \mathrm{CY} \cdot \mathrm{CY}_{1}}{\mathrm{AY}_{1} \cdot \mathrm{AY} \cdot \mathrm{CX}_{1} \cdot \mathrm{CX} \cdot \mathrm{BZ}_{1} \cdot \mathrm{BZ}}=\frac{\mathrm{AZ}_{1} \cdot \mathrm{BX}_{1} \cdot \mathrm{CY}_{1}}{\mathrm{AY} \cdot \mathrm{CX} \cdot \mathrm{BZ}} .
\end{gathered}
$$

But if $\mathrm{AD}, \mathrm{BE}, \mathrm{CF}$ are conjugate to the sides, $\frac{\mathrm{AZ}}{\mathrm{AY}}=\frac{\mathrm{AF}}{\mathrm{AE}}$, etc.

$$
\therefore \frac{\mathrm{AZ}_{1} \cdot \mathrm{BX}_{1} \cdot \mathrm{CY}_{1}}{\mathrm{AY} \cdot \mathrm{CX} \cdot \mathrm{BZ}}=\frac{\mathrm{AF} \cdot \mathrm{BD} \cdot \mathrm{CE}}{\mathrm{AE} \cdot \mathrm{BF} \cdot \mathrm{CD}}=1
$$


Therefore, by Carnot's theorem the six points $X, X_{1}, Y, Y_{1}, Z, Z_{1}$, lie on a conic.

Let $a_{2}, b_{2}, c_{2}$ be the radii of this conic parallel to the sides.

Then $\frac{\mathrm{AZ} \cdot \mathrm{AZ}}{\overline{\mathrm{AY}} \mathrm{Z}_{1} \cdot \mathrm{AY}}=\frac{c_{2}{ }^{2}}{b_{2}{ }^{2}} . \quad$ But $\frac{\mathrm{AZ} \cdot \mathrm{AZ}}{\mathrm{AY} Z_{1}, \mathbf{A Y}}=\frac{c \cdot \mathrm{AF}}{b \cdot \mathrm{AE}}=\frac{c_{1}{ }^{2}}{b_{1}{ }^{2}}$.

$$
\therefore \frac{c_{2}^{2}}{c_{1}^{2}}=\frac{b_{2}^{2}}{b_{1}^{2}}=\frac{a_{2}^{2}}{a_{1}^{2}}
$$

and the conic is homothetic with the circumconic and the centre is $K$.

$\mathrm{X}_{1} \mathrm{Y}_{1}, \mathrm{Y}_{1} \mathrm{Z}_{1}, \mathrm{Z}_{1} \mathrm{X}_{1}$ as also $\mathrm{XZ}, \mathrm{YX}, \mathrm{ZY}$ are conjugate to $\mathrm{BC}, \mathrm{CA}, \mathrm{AB}$. Therefore through the vertices of a triangle two triangles can be drawn with sides conjugate in reverse order to those of the first triangle and both have for "symmedian" or " cosine" centre the circumcentre of the triangle, etc.

$$
\frac{\mathrm{BX}}{a}+\frac{\mathrm{XX}_{1}}{a}+\frac{\mathrm{X}_{1} \mathrm{C}}{a}=1 \text {, }
$$

$\therefore$ since $\mathrm{Z}_{1} \mathrm{X}, \mathrm{X}_{1} \mathrm{Y}$ are equal and parallel to $\mathrm{Y}_{1} \mathrm{Y}, \mathrm{Z}_{1} \mathrm{Z}$

Also

$$
\frac{X_{1}}{a}+\frac{Y_{1}}{b}+\frac{Z Z_{1}}{c}=1
$$

$$
\frac{\mathrm{CX}_{1}\left(\mathrm{CX}_{1}+\mathrm{X}_{1} \mathrm{X}\right)}{a_{1}^{2}}=\frac{\mathrm{CY}\left(\mathrm{CY}+\mathrm{YY} \mathrm{Y}_{1}\right)}{b_{1}^{2}}
$$

$$
\therefore \frac{\frac{\mathrm{XX}_{1}}{a}+\frac{\mathrm{ZZ}_{1}}{c}}{\frac{\mathbf{a}_{1}^{2}}{a^{2}}}=\frac{\frac{\mathrm{ZZ}_{1}}{c}+\frac{\mathrm{YY}_{1}}{b}}{\frac{b_{1}^{2}}{b^{2}}} .
$$

Similarly

$$
\frac{\frac{Y Y_{1}}{b}+\frac{X X_{1}}{a}}{\frac{a_{1}^{2}}{a^{2}}}=\frac{\frac{Y Y_{1}}{b}+\frac{Z_{1}}{c}}{\frac{c_{1}^{2}}{c^{2}}} .
$$

From these 


$$
\frac{\frac{\mathrm{XX}_{1}}{a}}{\frac{b^{2}}{b_{1}^{2}}+\frac{c^{2}}{c_{1}^{2}}-\frac{a^{2}}{a_{1}^{2}}}=\frac{\frac{\mathrm{YY}_{1}}{b}}{\frac{c^{2}}{c_{1}^{2}}+\frac{a^{2}}{a_{1}^{2}}-\frac{b^{2}}{b_{1}^{2}}}=\frac{\frac{\mathrm{ZZ}_{1}}{c}}{\frac{a^{2}}{a_{1}^{2}}+\frac{b^{2}}{b_{1}^{2}}-\frac{c^{2}}{c_{1}^{2}}}=\frac{1}{\frac{a^{2}}{a_{1}^{2}}+\frac{b^{2}}{b_{1}{ }^{2}}+\frac{c^{2}}{c_{1}{ }^{2}}}
$$

Iet $\mathrm{A}_{2}, \mathrm{~B}_{2}, \mathrm{C}_{2}$ be the mid points of $\mathrm{XX}_{1}, \mathrm{YY}_{1}, \mathrm{ZZ}_{1}$. $\mathrm{KB}_{2}=\frac{1}{2} \mathrm{XY}$, and $\mathrm{XY}$ is parallel to $\mathrm{BE}$.

$$
\begin{aligned}
& \therefore \frac{\mathrm{XY}}{\mathrm{BE}}=\frac{\mathrm{CX}}{a}=\frac{\mathrm{CX}_{1}}{a}+\frac{\mathrm{X}_{1} \mathrm{X}}{a}=\frac{\mathrm{XX}_{1}}{a}+\frac{\mathrm{ZZ}_{1}}{c}=\frac{\frac{2 b^{2}}{b_{1}{ }^{2}}}{\frac{a^{2}}{a_{1}{ }^{2}}+\frac{b^{2}}{b_{1}{ }^{2}}+\frac{c^{2}}{c_{1}{ }^{2}}} . \\
& \therefore \mathrm{KB}_{2}=\frac{\frac{b^{2} \cdot \mathrm{BE}}{b_{1}^{2}}}{\frac{a^{2}}{a_{1}^{2}}+\frac{b^{2}}{b_{1}^{2}}+\frac{c^{2}}{c_{1}^{2}}}=\frac{b q \mathrm{~S}}{2 b_{1} \frac{a^{2}}{a_{1}^{2}}} \text {. } \\
& \therefore \frac{\frac{\mathrm{KA}_{2}}{p}}{\frac{a}{a_{1}}}=\frac{\frac{\mathrm{KB}_{2}}{q}}{\frac{b}{b_{1}}}=\frac{\frac{\mathrm{KC}_{2}}{r}}{\frac{c}{c_{1}}}=\frac{\mathrm{S}}{2 \Sigma \frac{a^{2}}{a_{1}^{2}}} \text {. }
\end{aligned}
$$

If $\mathrm{U}$ is the mid point of $\mathrm{BC}$ and $\mathrm{UE}_{1}$ is conjugate to $\mathrm{CA}$ then $\mathrm{KB}_{2} . \mathrm{UE}_{1}=\frac{1}{2} \mathrm{~KB}_{2} . \mathrm{BE}=\frac{q^{2} \mathrm{~S}}{8 \Sigma \frac{a^{2}}{a_{1}^{2}}}$.

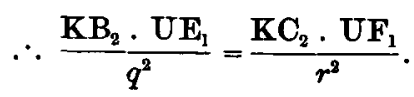

$\therefore \mathrm{G}$ and $\mathrm{K}$ are "isogonally conjugate."

If $\mathrm{T}$ is the pole of $\mathrm{XX}_{1}$ with regard to the "cosine" conic, the triangles $\mathrm{BOC}, \mathrm{X}_{1} \mathrm{TX}$ are similar.

$$
\therefore \frac{\mathrm{TA}_{2}}{\mathrm{OU}}=\frac{\mathrm{XX}_{1}}{a}=\frac{\frac{b^{2}}{b_{1}^{2}}+\frac{c^{2}}{c_{1}^{2}}-\frac{a^{2}}{a_{1}^{2}}}{\sum \frac{a^{2}}{a_{1}^{2}}}
$$




$$
\begin{aligned}
& \therefore \frac{\mathrm{TA}_{2}}{p}=\frac{\frac{a}{a_{1}}\left(\frac{b^{2}}{b_{1}{ }^{2}}+\frac{c^{2}}{c_{1}{ }^{2}}-\frac{a^{2}}{a_{1}{ }^{2}}\right)^{2}}{2 \mathrm{~S} \Sigma \frac{a^{2}}{a_{1}{ }^{2}}} . \\
& \mathrm{KT}=\mathrm{K} \mathrm{A}_{2}+\mathrm{A}_{2} \mathrm{~T}=\frac{2 p \frac{a^{2} b^{2} c^{2}}{a_{1}{ }^{2} b_{1}{ }^{2} c_{1}{ }^{2}}}{\mathrm{~S} \Sigma \frac{a^{2}}{a_{1}{ }^{2}}} .
\end{aligned}
$$

$\mathrm{KT} . \mathrm{KA}_{2}=p_{1}^{2}$ where $p_{1}$ is the radius of the "cosine" conic parallel to $p$.

$$
\therefore \text { the ratio of similarity } \frac{p_{1}}{p}=\frac{\frac{a b c}{a_{1} b_{1} c_{1}}}{\sum \frac{a^{2}}{a_{1}^{2}}} \text {. }
$$

Enough has been done to show the extensions and methods of proof. Considerations of space also prevent me adding investigations I have made on the extensions of Lemoine's, Tucker's, and Taylor's circles, on the Brocard points, on Inversion, etc. 